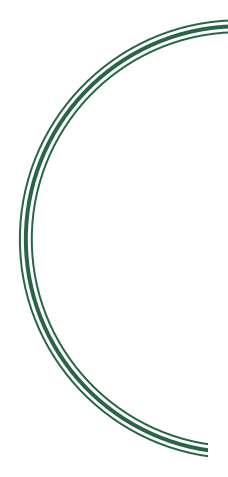

\title{
Mammalian Gut Microbiota and Immunity
}

\author{
Jean Kanellopoulos \\ Paris-Sud University, Orsay, France \\ Associate Editor of Biomedical Journal
}

$\mathrm{T}^{\mathrm{k}}$

This issue of Biomedical Journal includes two review articles on gut microbiota and the immune system. The enormous and recent interest in this scientific topic is attested by a large surge in publications. The first review article is written by Professor Vijay-Kumar and his colleagues and is entitled "Mammalian gut immunity" ${ }^{[1]}$ The second review article is written by Professor Lai and his colleagues with the title "Impact of the gut microbiota, prebiotics, and probiotics on human health and disease". ${ }^{[2]}$

Professor Vijay-Kumar's review article describes the tremendous complexity of the mucosal immune system, which has the ability to eliminate pathogens while tolerating the commensal bacteria needed for the production of nutrients, among other functions. The authors describe the role of the intestinal epithelium as a physical and chemical barrier. The epithelium also consists of cells capable of secreting numerous cytokines and chemokines that modulate the chemotaxis of various hematopoietic cells. The role of intestinal macrophages, dendritic cells, T lymphocytes, and innate lymphoid cells is also analyzed. The impact of microbiota and bacterial metabolites on the development of the host mucosal immune system is described.

The second article, by Professor Lai, summarizes recent studies on gut microbiota and their potential role in several human diseases such as obesity and liver disease, and lung immunity. The role of microbiota on nutrient metabolism and fermentation products is analyzed, and the production of beneficial or harmful compounds by intestinal bacteria is critical for maintaining host homeostasis. Interestingly, in the section on prebiotics, the authors summarize their unpublished results on prebiotics found in fungi that are used in traditional Chinese medicine.

As gut microbiota are featured prominently in both articles, it is tempting to speculate that after diverging for many centuries, Eastern and Western science may be converging again through the unexpected role played by gut microbes in immunity, health, and disease.

\section{REFERENCES}

1. Chassaing B, Kumar M, Baker MT, Singh V, Vijay-Kumar M. Mammallian Gut Immunity. Biomed J 2014;37:246-58.

2. Lin CS, Chang CJ, Lu CC, Martel J, Ojcius DM, Ko YF, et al. Impact of the gut microbiota, prebiotics, and probiotics on human health and disease. Biomed J 2014;37:259-68. 